\title{
The Diagnostic Gap in Colorectal Cancer
}

\author{
Amandeep Kaur, Joseph Salhab, Kanza Soomro and Javier Sobrado* \\ Larkin Community Hospital, South Miami, FL, USA \\ *Corresponding author: Sobrado J, Gastroenterology Fellow, Larkin Community Hospital, South Miami, FL, USA, Tel: 3052700402; E-mail: \\ jsobrado@gastromedmiami.com
}

Received date: December 20, 2017; Accepted date: December 26, 2017; Published date: December 31, 2017

Copyright: @ 2017 Kaur A, et al. This is an open-access article distributed under the terms of the Creative Commons Attribution License, which permits unrestricted use, distribution, and reproduction in any medium, provided the original author and source are credited.

\section{Introduction}

Colorectal cancer is the third leading cause of cancer-related deaths in women and the second leading cause in men within the United States. In 2017 alone, there were approximately 135,430 new cases of colorectal cancer (CRC) and about 50,260 deaths attributable to CRC [1].

Most CRC begins as a small growth, referred to as "polyps" that reside on the inner lining of the colon or rectum. Certain types of polyps, called "adenomatous polyps" are considered precancerous while hyperplastic and inflammatory polyps typically do not increase cancer risk [2]. These precancerous polyps can eventually transform into cancer over the course of several years [2]. One of the reasons the death rate from CRC has been dropping over the past several decades has been our increased efforts to convince patients to undergo screening colonoscopies, which allow precancerous colorectal polyps to be removed before they can develop into cancer. In addition, CRC is also being found earlier when the disease is easier to treat as compared to previous years.

The American Cancer Society (ACS) guidelines recommend that men and women of average risk for developing colon cancer should begin screening at age $50[3,4]$. According to the ACS, there are approximately 23 million people between the ages of 50-75 that have not been screened for CRC. Per the census bureau, approximately 10,000 people turn 50 years of age each day, which also adds to the population that requires initial screening [5]. Our most accurate population statistics stem from the year 2012, where only $65 \%$ of population greater than 50 years of age were screened, leaving approximately $1 / 3$ of the at risk population unscreened [4].

According to the National Interview Survey (NHIS) and the Behavioral Risk Factor Surveillance System (BRFSS), only one-half of the survey respondents ages 50 years or older have been screened as recommended with Fecal Occult Blood Testing (FOBT) and endoscopy (sigmoidoscopy/colonoscopy) [6]. According to Seeff et al. [7] screening rates for CRC remain low compared with screening rates of other cancer. Of the 70.1 million persons in the United States aged 50 years or older at average risk for CRC, 28.3 million people (40.4\%) have been screened within recommended intervals, 15.7 million with FSIG or colonoscopy only, 6.7 million with FOBT only, and 5.9 million with both FOBT and endoscopy [7]. The remaining 41.8 million people have not been screened for CRC [7].

This disparity in CRC screening has led us to introduce the term known as the "Diagnostic Gap" or DG. The DG is defined as "the incidence of CRC in the unscreened population that we fail to identify in its early stages due to lack of compliance with screening" [6]. Due to their delay in diagnosis, these individuals have an overall poorer prognosis . Per the survival data from the SEER database, the 5 years survival rate for those diagnosed as Stage I is significantly higher than those diagnosed at an advanced stage $-90.1 \%$ vs. $13.1 \%$ [7].

Therefore, the biggest question that remains is how we will eliminate the tumor burden and begin closing the DG. Screening the entire population over 50 years of age with only colonoscopies is challenging due to lack of physician training, manpower needed, and patient compliance. The biggest reason people are apprehensive to undergoing a colonoscopy is that they believe the test is difficult or painful, they don't like the preparation, they don't have insurance, and they are concerned about other factors, including taking time off from work, getting a ride home, and high out-of-pocket expenses. Colonoscopy demand ranges from 2.21 to 7.96 million per year [8]. To screen people with colonoscopy at least once at the age of 65 years, we would need an additional 1360 gastroenterologists [8]. An additional 32,700 gastroenterologists would be required for colonoscopies every 10 years thereafter [8]. Based on Seeff et al. [7] study, the number of tests needed for screening varied based on screening program. A program based on current screening patterns would require about 16 million sigmoidoscopies and colonoscopies; whereas, a program using FOBT only would require 1 million colonoscopies as a diagnostic follow up [7]. Programs using FSIG would require $>40$ million sigmoidoscopies and approximately 2-3 million diagnostic colonoscopies [7].

According to Seeff et al. [7] there is an available capacity of 6.7 million flexible sigmoidoscopies and 8.2 million colonoscopies. Sufficient capacity exists to screen the unscreened population within 1 year using FOBT followed by diagnostic colonoscopy for positive results. Depending on the proportion of available capacity used for CRC screening, it could take up to 10 years to screen the unscreened population using flexible sigmoidoscopy or colonoscopy [7]. The capacity for widespread screening with FOBT does exist whereas screening with FSIG or colonoscopy depends on the proportion of available capacity used for CRC screening [7].

On the other hand, people may be screened for CRC via FOBT or multi-target stool DNA testing. The tests are done at home, with no colon preparation, pain, discomfort or having to change their schedule. The best test for that right now, with the highest accuracy, uses stool DNA to detect CRC and precancerous polyps. Only those with positive tests would then require a follow up colonoscopy screening [5]. This would allow for us to screen a larger population in the shorter amount of time along with reducing the colonoscopy screening burden. Of note, this method is limited to population with a standard risk of CRC and excludes high risk population such as those with family history of CRC, personal history of CRC, history of polyps, those with IBD, and those with familial diseases. The high risk population will follow the standard guidelines for CRC screening via colonoscopy. "The incidence of CRC via flexible sigmoidoscopy is $1.29 \%$ in the screened and $1.64 \%$ in the unscreened group" [6]. If we can close this gap and screen $100 \%$ 
Citation: Kaur A, Salhab J, Soomro A, Sobrado J (2017) The Diagnostic Gap in Colorectal Cancer. J Oncol Transl Res 3: 125. doi:

Page 2 of 2

of those 23 million people between the ages of 50-75 years who have never underwent evaluation for CRC, we will diagnose approximately 377,200 new colon cancers. Furthermore, the number-needed-toscreen to avert one CRC diagnosis and one CRC related death is 278 and 850 , respectively [6]. Therefore, if we screened $100 \%$ of the 23 million people, we would avert approximately 82,733 CRC diagnosis and 27,058 CRC related deaths. Each day, 10,000 new people turn 50 years of age. By screening $100 \%$ of this population, we will diagnose 164 CRC, avert 35 new CRC diagnoses, and prevent 11.8 CRC related deaths each day. These numbers are staggering when you consider the sheer number of individuals walking around with CRC, harboring the cancer, and eventually being diagnosed at a later stage. As a result of our increased efforts in screening for CRC, there are more than 1 million survivors of CRC in the United States [2]. However there is still much work left to be done. We believe that by closing the DG, we could save more than 25,000 lives by 2025 .

\section{References}

1. https://fightcolorectalcancer.org/prevent/about-colorectal-cancer/factsstats/
2. https://www.cancer.org/cancer/colon-rectal-cancer/about/what-iscolorectal-cancer.html

3. (2011) Closing the gap in colon cancer screening.

4. Carrie NK, Djenaba AJ, Jessica BK, Arica W, Marcus P (2013) Vital signs: Colorectal cancer screening test use-United States, 2012. Centers for Disease Control and Prevention.

5. https://www.census.gov/topics/population/about.html

6. Kaur A, Salhab J, Sobrado J (2016) Recognizing diagnostic gap in colorectal cancer. Intern Med 6: 1-5.

7. Seeff LC, Manninen DL, Dong FB, Chattopadhyay SK, Nadel MR, et al. (2004) Is there endoscopic capacity to provide colorectal cancer screening to the unscreened population in the united states? Gastroenterology 127: 1661-1669.

8. Vijan S, Inadomi J, Hayward RA, Hofer TP, Fendrick AM (2004) Projections of demand and capacity for colonoscopy related to increasing rates of colorectal cancer screening in the United States. Aliment Pharmacol Ther 20: 507-515. 